\title{
Algae and Their Vital Importance in Life
}

\author{
Latife Ceyda Irkin \\ Canakkale Onsekiz Mart University, School of Applied Science, \\ Department of Fisheries Technology, Canakkale-Turkey \\ E-mail: latifeirkin@gmail.com
}

\begin{abstract}
The emergence of life started with biological evolution after the conditions have become appropriate for the creation of the earth and the survival of living things. The formation of sun-absorbing compounds (pigment) has led to the formation of high autotrophic plants as well as microscopic and macroscopic algae for capable of photosynthesis. Algae, which enter into both aquatic and terrestrial areas of the world with their members, are not only the producers of raw material source, but also have an important function as a major oxygen source. Algae have been used by people in every period of history. However, the first original work on the use of algae was compiled by Emperor Shen Nung in 2700 B.C.

Today, most of the seaweed is used as food. $50 \%$ of the algae are collected and cultivated for economic purposes in the world that are used in food industry, $40 \%$ of the algae are used in pharmaceutical and cosmetic industry and $10 \%$ algae are used in other fields. Algae contain important polysaccharides and bioactive compounds. Of these, extracts such as agar, carrageenan and alginate are among the products that have no alternatives in many industries today. Approximately $45 \%$ of algae are produced or collected to obtain alginate, carrageen, agar and other phicocolloids.
\end{abstract}

Keywords: Algae, bioactive compounds, life, food and pharmaceutical industry.

DOI: $10.7176 /$ JSTR/5-9-13

\section{Introduction}

The formation of sun-absorbing compounds has led to the formation of high autotrophic plants as well as microscopic and macroscopic algae that are capable of photosynthesis. Firstly, prokaryotic organisms such as bacteria and Cyanobacteria, which are the first step of the plants, were developed. Then, eukaryotic microscopic algae emerged from the nuclei separated by a prominent wall, colony-forming algae with filamentous and thallus developed. Algae play a very important role from the begining of the life. Algae, which enter all areas of our life with their members living on both land and water, are not only the producers of the raw material source of tons of products consumed each year, but also have an important function as a major oxygen source. Algae, which started to give oxygen to the atmosphere nearly 3.5 billion years ago, also contributed to the formation of land.

There are also those that directly accumulate lime in their bodies and indirectly contribute to rock formation. These rocks, called stromatolites, consist of interactions of algae and sand mixtures lasting for billions of years which have an average growth of $1.5 \mathrm{~mm}$ per year, have been found with the remains of oxygen-producing organisms 3.5 billion years ago. They also contribute coastal formation, especially with calcareous algae, such as Corallinales members.

\section{The General Usage of Algae}

Algae have been used by people for a very long time. However, the first original record for the use of algae was compiled by Emperor Shen-Nung in 2700 BC. The work states that seaweed was used as medicine and foodstuff in Far East countries.

It has been known that algae has been used as food in Far Eastern countries and as fertilizer in European countries since Middle age. Later, during the Roman Empire algae were used as a color substance in the cosmetic industry. But the important economic exploitation of algae was the years of the 1670s, first started by the Chinese and the Japanese.

In the 17 th century, the talluses of algae were burned and mixtured with sodium-potassium salts to use in glass making. Although today developing industry has succeeded in synthetic production of many organic compounds, some compounds are still obtained directly from algae. Especially agar-agar and alginate appear as algae products which are used in different sectors in the world markets. The economic

99 | $\mathrm{P}$ a g e

www.iiste.org 
benefits of algae are a real resource for small countries. America, Japan, China, India, Northern Europe and England are the leading countries. In these countries, the expression "algae cultivation" is a substitute expression.

There are species used as indicators of pollution. By taking the metals from water into their bodies, they contribute to clean the water and maintain the vitality by producing plenty of oxygen with photosynthesis. It is known that $70 \%$ of the oxygen in the atmosphere is produced by aquatic plants. There are also indicator species (Batrachospermum $s p$ ) are used to determine drinking water quality. If some species such as Phaeocystis sp. are overgrown and shaken by waves, the matrix polysaccharides of the species become soap bubbles. These foams, which can be annoying and sometimes entertaining to tourists, can drift into the sea, form colonies or block fishing nets. The overproduction of Chrysochromulina polylepis was observed in May 1988 around the southern coast of Norway and the western coast of Sweden. This flowering has been quite toxic to fish and even marine algae. It has caused more damage especially for fish farms where salmon species is grown.

Planktonic haptophytes such as Emiliania huxleyi and Phaeocystis pouchetii produce reduced dimethyl sulfide (DMS) produce compounds. DMS is volatile and released into the atmosphere, where they are photochemically converted into small droplets of sulfuric acid that serve as nuclei in cloud formation. For this reason, planktonic haptophytes, which grow occasionally by forming large blooms, contribute significantly to the acidity of rainwater and affect the climate (Figure 1,2).

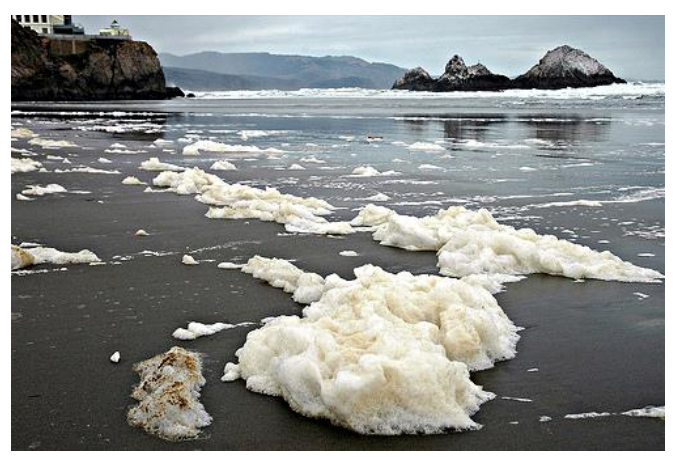

Figure 1. Sea foam (Phaeocystis sp.)

(https://ww2.kqed.org/quest/2011/05/02/sea-foam-lathers-up-the-ocean/)

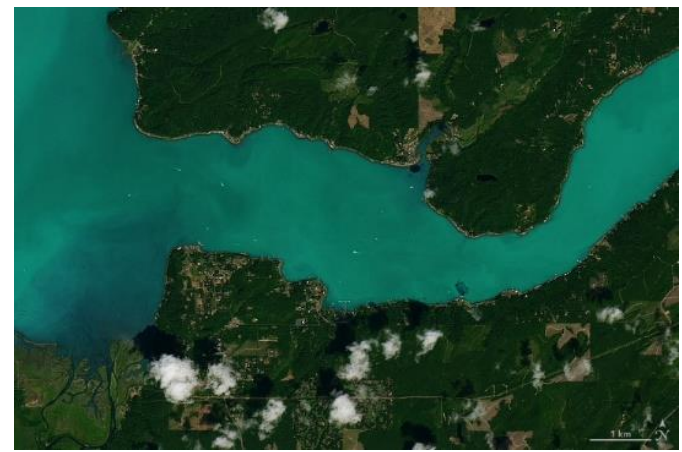

Figure 2. Bloom of Emiliania huxleyi

(https://earthobservatory. nasa. gov/images/90679/plankton-abloom)

\section{Some Bioactive Compounds of Algae}

Algae contain harmful toxic compounds as well as products used in different branch of industry as raw materials. Among these, polysaccharides such as mannitol, laminarine, fucoidan, alginate, agar agar, carragen, hypnean and phylloporane are among the algae (Venugopal, 2019).

Mannitol is a polysaccharid found in the core water of brown seaweeds. It replaces free sugar in land plants. Mannitol is a naturally occurring polyol that can protect organisms against environmental stress. Mannitol biosynthesis in algae, identifying how their twisting evolutionary history, has led to diverse and unexpected combinations of mannitol metabolic enzymes in these organisms. Mannitol is used as a sweetener as well as a medication for decreasing pressure in the eyes and brain. This important compound can comprise up to $25 \%$ of the dry weight of some algal species, and this work could have implications for enhancing mannitol production in the future. . It has mild diarrhea effect when taken in high doses (Tonon et al., 2016). 
Laminarin, also called laminaran, a principal reserve polysaccharide of brown seaweed, is an active component that is extracted and isolated from the dry thallus of Phaeophyta species contains large amount of neutral sugars with low concentration of uronic acid, like Laminaria japonica, Ecklonia kurome, or Eisenia bicyclis (Deleris et al., 2016). Laminarin is a water-soluble polysaccharide that consists of $\beta-(1$ 3 )-glucan with $\beta$-(1-6)-linkages of 20-25 units (Nelson and Lewis, 1974). The molecular weight of laminarin is lower than other polysaccharides present in seaweed and can be separated using dialysis with different molecular weight cut-off membranes. Thus laminarin contributes to the dietary fiber intake and plays a role in cancer prevention. Laminarin has also been proposed for its antitumor effect by some researchers (Deleris et al., 2016).

Fucoidan is a sulfated polysaccharide (MW: average 20,000) found mainly in various species of brown algae and brown seaweed such as mozuku, kombu, bladderwrack, wakame, and hijiki (variant forms of fucoidan have also been found in animal species, including the sea cucumber) (Atashrazm et al., 2015). Fucoidan is important in cosmeceutical and food production as it is possible to extract from cheap resources and used in novel drugs and functional food production. In addition, it is useful in antiviral, anticoagulant, and antibacterial activities. (Lee et al., 2004). In Asian countries such as China, Japan, and Korea, seaweeds are considered as dietary supplements and also are reported to have been used for boosting up the immune system. Mekabu (sporophyll of Undaria pinnatifida), a dietary alga, is reported to exert antitumor activity and enhance the immune response. Several biological activities were reported for fucoidan. Some authors showed that fucoidan affects the secretion of extracellular matrix proteins influences cell proliferation (Koyanagi et al., 2003), and can induce apoptosis (Aisa et al., 2005).

Alginate was first described by the British chemist Stanford in 1881, and exists as the most abundant polysaccharide in the brown algae comprising up to $40 \%$ of the dry matter. (Draget, 2009). It is located in the intercellular matrix as a gel containing sodium, calcium, magnesium, strontium and barium ions (Haug, 1964). Alginate is widely used in industry because of its ability to retain water, and its gelling, viscosifying and stabilising properties. Several bacteria also produce alginate (Linker and Jones, 1964; Gorin and Spencer, 1966), and Azotobacter vinelandii has been evaluated as a source for industrial production. All commercial alginates stem from algal sources especially Brown algae like Laminaria, Macrocystis, Ascophyllum, Eclonia, Lessonia, Durvillea, and Sargassum sp. Alginate extraction is based on conversion of all insoluble salts of alginic acid that are present within the cell wall of brown seaweed to the soluble $(\mathrm{Na}+)$ salt of alginic that is subsequently recovered as alginic acid or calcium alginate. (Featherstone, 2015). Alginate is used in various doses and mixed with other substances to slim down without fasting. This low calorie gel thickens the stomach acid of the wearer, resulting in a feeling of fullness. They are used in the production of calorie-free food that is specially produced for diet or diabetes by adding various aroma and calorie-free taste substances. In recent years, alginate has been used in nanotechnology applications for drug release into the body by forming natural polymer based microspheres.

Agar agar is a polysaccharide obtained by extracting red algae. These seaweeds are mostly Gelidium and Gracilaria species and are collected from different countries. Agar is not a single chemical substance, it is also a polymer composed of Agarose and Agaropectin polysaccharides. The thin layer agar is in powder form. Features that differentiate the quality; factors such as gel strength, degree of gelling and melting, color, solubility change the quality of agar. Generally, the gelling temperature of the agar is cooled to a hot solution of $30-40^{\circ} \mathrm{C}$ to obtain gelling. It melts when heated above $90-95^{\circ} \mathrm{C}$. It has the ability to draw up to twenty times the weight of the agar. Agar is used in food industry, bakery, confectionery, milk and dairy products and meat products. In Food Industry; heat-resistant gel formation and a different melting temperature and gel formation temperature are preferred for particular uses. For this purpose, it is used as a stabilizer in cookies, puddings, decoration jellies, pastry fillings, cream pastries and other similar foods (Saha and Bhattacharya, 2010). Use 0.2-0.5\% in cake decorations (sugar coatings and creams) to increase stability. And it prevents the sugared liquid from flowing and sticking to the package. Its use of $0.12 \%$ ensures consistency and homogeneity in sherbet and ice cream. It can also be used to improve the structure and slicing quality of cheeses. Agar is practically the most widely used microbiology as a culture medium for all pathogenic and non-pathogenic bacteria. This use is related to the strong gel properties, flexibility, clarity and stability of the agar (Draget, 2000). Materials used for dental applications; It is used to accurately mold prosthetic objects. By mixing agar with different substances, precise results are obtained in dental applications. The concentration of agar in the effect materials is $13-17 \%$. (http://www.vankim.com/productsr/thickeners/agar-agar).

Carrageenans were introduced on an industrial scale in the 1930s, they were first used in China around 600 B.C. (where Gigartina was used) and in Ireland around 400 A.D (Loureiro et al., 2017). Since the late 1960 s, there's been controversy surrounding the health effects of carrageenan. Some evidence suggests that carrageenan triggers inflammation, gastrointestinal ulcerations, and that it

101 | P a g e

www.iiste.org 
damages your digestive system. People have been petitioning for products with carrageenan to be labeled with a warning or removed entirely. Carrageenan are a family of linear sulfated polysaccharides that are extracted from red edible algae. They are widely used in the food industry for their gelling, thickening, and stabilizing properties. Their main application is in dairy and meat products, due to their strong binding to food proteins. Carrageenan has a variety of uses. Though it has no flavor or nutritional value, it is a useful thickening agent and stabilizer. Some manufacturers include it in products like chocolate milk, to stop the milk from separating. It can also substitute for fat in non- or low-fat foods and dairy replacement products aimed at the vegan market. Some manufacturers use carrageenan as a binder in processed deli meats. Others inject it into pre-cooked poultry to tenderize the meat and keep it juicy for longer. Companies often use carrageenan as a vegan alternative to gelatin, in desserts, for example. It is also a common ingredient in canned pet food. Non-food products, such as air freshener gels and toothpaste also frequently contain carrageenan. The Food and Drug Administration requires manufacturers to state whether products contain carrageenan on labeling. (https://www.medicalnewstoday.com/articles/323117.php). There are three main varieties of carrageenan, which differ in their degree of sulfation. Kappa-carrageenan has one sulfate group per disaccharide, iota-carrageenan has two, and lambda-carrageenan has three. Gelatinous extracts of the Chondrus crispus (Irish moss) seaweed have been used as food additives since approximately the fifteenth century. Carrageenan is a vegetarian and vegan alternative to gelatin in some applications or may be used to replace gelatin in confectionery (FAO, 2011).

Hypnean is is the extract of Hypnea musciformis and other Hypnea species. Its chief value is its strong gelling ability. Furthermore, the physical properties of hypnean gels can be controlled to a considerable extend. Therefore, in as much as Hypnea species are rather plentiful throughout the world, hypnean could become an extract of economic importance (Whistler, 1973).

\section{Conclusion}

According to FAO, aquaculture production in the world has reached to 14.8 million tons and total production amount (in terms of wet weight) has reached to 16.9 million tons (2005). While this amount was 10.6 million tons in 2011, it increased to 11.6 million tons in 2012. Compared to other countries, China ranks first in the production of aquatic plants with 8.8 million tons and constitutes $71.1 \%$ of the total production. Total aquatic plant production reached 30.1 million tons (FAO, 2016). Most of this production is carried out by China and India.

Most of the collected seaweed today is used as food. $50 \%$ of collected and cultivated algae in the world are used in food industry, $40 \%$ of algae are used in pharmaceutical and cosmetic industry and $10 \%$ in other fields. Algae contain important polysaccharides in their bodies. Of these extracts such as agar, carrageenan and alginate are among the products that have no alternatives in many industries today. Approximately $45 \%$ of algae are produced or collected to obtain alginate, , agar and phycocolloids.

The needs and necessities starts due to the increasing population density and changing climatic conditions, people seem to benefit more and more from the aquatic resources. As an important protein source with a wide range of utilization, algae have attracted researchers more and more in recent years. Further studies are encouraged on the utilization and production of algaa as food ingredients or raw materials in various fields.

\section{References}

Aisa, Y. Et al. (2005). Fucoidan Induces Apoptosis of Human HS-Sultan Cells Accompanied by Activation of Caspase-3 and Down-regulation of ERK Pathways. American Journal of Hematology. 78 (1), 7-14. doi: 10.1002/ajh.20182

Atashrazm, F. et al. (2015). Fucoidan and Cancer: A Multifunctional Molecule with Anti-Tumor Potential. Marine Drugs. 13 (4), 2327-2346. doi:10.3390/md13042327

Dipjyoti, S., \& Bhattacharya, S. (2010). Hydrocolloids as Thickening and Gelling Agents in Food: a Critical Review. Journal of Food Science Technology. 47(6), 587-597. doi: $10.1007 / \mathrm{s} 13197-010-0162-6$

Draget, K. I., Go, P., \& Williams, P. A. (2000). Alginates. Handbook of hydrocolloids. New York: Woodhead Publishing Ltd. 379-393.

FAO, 2005. Overview of Fish Production, Utilization, Consumption and Trade. Fishery.

102 | P a g e

www.iiste.org 
FAO, 2011. Agar and Carrageenan Manual. Fao.org (1965-01-01). Retrieved on 2011-12-10.

FAO. 2016. The State of World Fisheries and Aquaculture 2016 - Meeting the sustainable development goals, Rome. Licence: CC BY-NC-SA 3.0 IGO.

Gorin, P. A. J., \& Spencer, J. F. T. (1966). Exocellular Alginic Acid from Azotobacter vinelandii. Canadian Journal of Chemistry. 44 (9), 993-998.

Haug, A. (1964). Composition and Properties of Alginates. Norwegian Institute of Seaweed Research, 30.

Draget, K. I. (2009). Handbook of Hydrocolloids. (2nd edition). Woodhead Publishing, 807828.

Koyanagi, S. et al. (2003). Oversulfation of Fucoidan Enhances its Anti-angiogenic and Antitumor Activities. Biochemical Pharmacology. 65(2), 173-179. doi: 10.1016/s00062952(02)01478-8

Lee, J. B. et al. (2004). Novel Antiviral Fucoidan from Sporophyll of Undaria pinnatifida (Mekabu). Chemical and Pharmaceutical Bulletin. 52(9), 1091-1094. doi: $10.1248 / \mathrm{cpb} .52 .1091$

Linker A \& Jones RS, 1964, A polysaccharide resembling alginic acid from a Pseudomonas micro-organism, Nature, 204,187-188.

Nazimah, H. et al. (2015). Seaweed minor constituents. Seaweed Sustainability: Food and NonFood Applications, 193-242. Elsevier Inc. (Chapter 8). doi:10.1016/B978-0-12-418697$2.00008-8$

Nelson, T. E., \& Lewis, B. A. (1974). Separation and characterization of the soluble and insoluble components of insoluble laminaran. Carbohydrate Research. 33, 63-74.

Déléris, P., Nazih H., \& Bard, J. M. (2016). Seaweeds in Human Health. (1st edition). Seaweed in Health and Disease Prevention. 10, 319-367. doi: 10.1016/b978-0-12-802772-1.00010-5

Rafael, R. et al. (2017). Applications of Carrageenan: with Special Reference to Iota and Kappa Forms as Derived from the Eucheumatoid Seaweeds. Tropical Seaweed Farming Trends, Problems and Opportunities: Focus on Kappaphycus and Eucheuma of Commerce. Berlin, Germany: Springer International Publishing. 165-171.

Featherstone, S. (2015). Processing Procedures for Canned Food Products. (14th Edition). A Complete Course in Canning and Related Processes. Woodhead Publishing.

Tonon, T., Li, Y., \& McQueen-Mason, S. (2016). Mannitol Biosynthesis in Algae: More Widespread and Diverse than Previously Thought. New Phytologist. 213(4), 1573-1579. doi: 10.1111/nph.14358

Venugopal, V. (2019). Sulfated and Non-Sulfated Polysaccharides from Seaweeds and their Uses: An Overview. EC Nutrition 14(2), 126-141.

Whistler, R. 1973. Industrial gums: Polysaccharides and Their Derivatives. (2nd edition). New York: Academic press.

http://www.vankim.com/products/thickeners/agar-agar

https://www.medicalnewstoday.com/articles/323117.php

https://ww2.kqed.org/quest/2011/05/02/sea-foam-lathers-up-the-ocean/

https://earthobservatory. nasa. gov/images/90679/plankton-abloom 